\title{
Development of Tailless Two-winged Flapping Drone with Gravity Center Position Control
}

\author{
Hiroto Nagai, ${ }^{1 *}$ Kazutaka Nakamura, ${ }^{2}$ Koki Fujita, ${ }^{3}$ Issei Tanaka, ${ }^{4}$ \\ Shuji Nagasaki, ${ }^{4}$ Yutaka Kinjo, ${ }^{4}$ Shintaro Kuwazono, ${ }^{1}$ and Masahiko Murozono ${ }^{3}$ \\ ${ }^{1}$ Nagasaki University, 1-14 Bunkyo-machi, Nagasaki 852-8521, Japan \\ ${ }^{2}$ Murata Manufacturing Co., Ltd., 1-10-1 Higashikotari, Nagaokakyo-shi, Kyoto 617-8555, Japan \\ ${ }^{3}$ Nippon Bunri University, 1727 Ichigi, Oita 870-0397, Japan \\ ${ }^{4}$ Kyushu University, 744 Motooka, Nishi-ku, Fukuoka 819-0395, Japan
}

(Received December 14, 2020; accepted January 8, 2021; online published January 26, 2021)

Keywords: flapping wing, micro air vehicle, autonomous flight, biomimetics, flexible structure

We have developed a tailless, two-winged flapping drone with a full span length of $180 \mathrm{~mm}$ and a total weight of $20.5 \mathrm{~g}$. The developed flapping drone is characterized by three biomimetic techniques: an anisotropic vein pattern reinforcing the wing surfaces, an elastic flapping mechanism, and gravity center position control in the abdomen. On the basis of experimental and numerical results, the flapping wings are reinforced by a vein pattern made of an anisotropic carbon fiber-reinforced plastic (CFRP) laminate to passively provide appropriate aeroelastic deformation and positively utilize snap-though buckling on the wing surface at stroke reversals to provide a fast feathering rotation. The flapping wing kinematics are provided by a novel flapping mechanism with an energy recovery system using the elasticity of the mechanical system. Unlike other previously developed flapping robots, feedback control to stabilize the pitch and roll angles of the drone's body is conducted using a technique of gravity center position control, where the tail angles of the body are changed similarly to the abdominal movements of insects in flight. The developed flapping drone has succeeded in an autonomous hovering flight for more than $30 \mathrm{~s}$ and a vertical take-off under a wireless condition with the gravity center position control.

\section{Introduction}

Flapping-wing-type micro air vehicles (FMAVs), which are inspired by the flight of birds and insects, are characterized by a lightweight body and flexible flapping wings with complex wing motions. These characteristics contribute to high flight maneuverability, high safety in terms of collision with objects, and low noise compared with the conventional rotary-wingtype drones. Hence, FMAVs are regarded as human/nature-friendly drones with great potential in various applications for people, animals, and plants, such as in disaster relief, agriculture,

*Corresponding author: e-mail: nagai.hiroto@nagasaki-u.ac.jp https://doi.org/10.18494/SAM.2021.3222 
education for children, maintenance checks of plants and equipment, and urban security. Recently, a few FMAVs with a handheld size have succeeded in autonomous hovering flight. The "Nano hummingbird" developed by AeroVironment, Inc., which has a span length of $160 \mathrm{~mm}$ and a weight of $19 \mathrm{~g}$, is one of the most successful FMAVs. ${ }^{(1)}$ The Nano hummingbird has been followed by several other successful FMAVs ["Colibri" with a span length of $210 \mathrm{~mm}$ and a weight of $22 \mathrm{~g}^{(2)}$ and "KUbeetle-S" with a span length of $170 \mathrm{~mm}$ and a weight of $16.4 \mathrm{~g}^{(3)}$. These flapping drones have succeeded in autonomous stable hovering flight, in which they controlled the aerodynamic forces generated by the two flapping wings by changing the support conditions of the two wing bases. The "Delfly Nimble" FMAV with a span length of $330 \mathrm{~mm}$ and a weight of $28.2 \mathrm{~g}$ is a tailless, four-winged flapping robot and has succeeded in rapid banked turns by changing the flapping frequencies of the right and left wings and modulating the wing base supports. ${ }^{(4)}$ However, these successful flapping robots have not yet attained a comparable flight performance to birds and insects in terms of a lightweight body, flight duration, agile flight, structural durability, and low noise. The technologies employed by birds and insects remain largely unutilized in flapping robots. According to observations in previous studies, ${ }^{(5,6)}$ some insects change their abdominal angles during flight. Dyhr et al. ${ }^{(7)}$ experimentally confirmed that the movement of a hawkmoth's abdomen was synchronized with the changes in its flight and reported that the abdominal movement contributes to the flight stability by modulating the center of gravity (C.G.) of the body and by changing the aerodynamic force direction. However, no flapping drone has yet employed the technique of gravity center position control in its autonomous flight.

In this study, we have developed a flapping drone, which is a tailless, two-winged, autonomous flying robot with a full span length of $180 \mathrm{~mm}$ and a body weight of $20.5 \mathrm{~g}$. Autonomous hovering flight and vertical take-off are conducted with a technique of gravity center position control. The developed flapping drone is characterized as follows:

(1) The lightweight flapping wings are reinforced by an anisotropic vein pattern to provide both adequate strength and appropriate aeroelastic deformation during flapping. In addition, snap-through buckling of the wing surface is positively utilized to enhance the aerodynamic efficiency by producing a faster feathering rotation at stroke reversals.

(2) The flapping wings are actuated by a novel flapping mechanism to convert a rotation to a reciprocating motion. The flapping mechanism based on a crank-slider linkage with elastic supports has an energy recovery system that uses the elasticity of the mechanism.

(3) Feedback control to stabilize the pitch and roll angles of the body is conducted with a technique of gravity center position control. The C.G. of the body is modulated by changing the mounting angles of a battery at the tail of the drone similarly to the abdominal movements of insects.

\section{Specifications of Flapping Drone}

The photograph and schematic diagram of the developed flapping drone are shown in Fig. 1. The specifications of the drone are tabulated in Table 1 . The full span length is $180 \mathrm{~mm}$ and the 


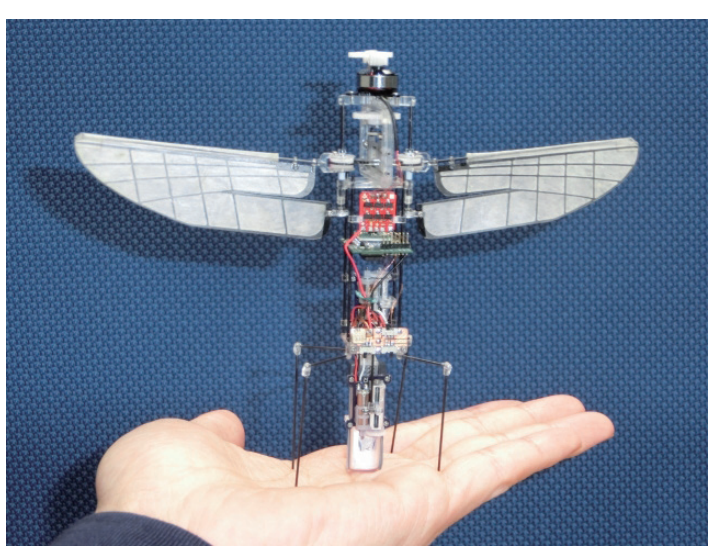

(a)

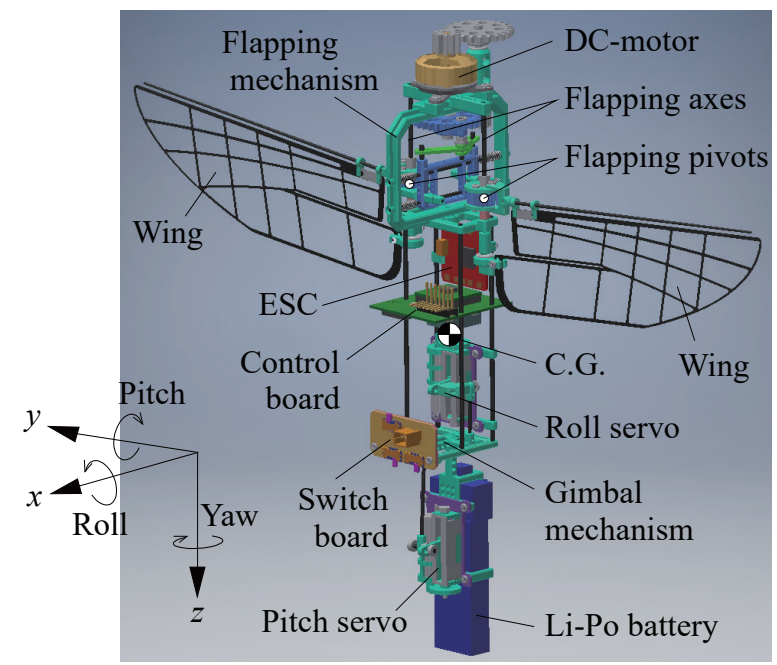

(b)

Fig. 1. (Color online) Photograph and schematic diagram of flapping drone.

total mass of the drone is $20.5 \mathrm{~g}$. A three-dimensional coordinate system fixed with the body is defined with the origin at the C.G. of the drone in the neutral positions of the flapping wings and battery. The $z$-axis is directed from the anterior to the posterior of the body, the $x$-axis is directed from the dorsal to the ventral, and the $y$-axis is directed to the right wing in the neutral flapping position. The C.G. is located $24.0 \mathrm{~mm}$ downward from the center of the two flapping pivots. The two flapping wings are driven by a brushless DC motor (AP03-7000, HobbyKing. com). The rotational motion generated by the motor is converted to a flapping motion in the $x-y$ plane around the flapping axes by the flapping mechanism described in Sect. 3. The flapping motion applied to the wing bases induces a passive aeroelastic deformation of the wings during a flapping cycle. Consequently, the wings passively obtain an appropriate combination of flapping and feathering motions and generate lift in the $-z$-direction. The electric power is supplied by a lithium polymer (Li-Po) battery (XP01101ES, $110 \mathrm{mAh}, 1 \mathrm{~S}, 15 \mathrm{C}$, Dualsky.com), which is mounted at the tail of the drone. The mounting angles of the battery can be modulated by two linear servo actuators to change the gravity center position in the $x$ - and $y$-directions to stabilize the pitch and roll angles of the body (described in Sect. 5). The yaw control of the drone is dependent on passive stability because the drone is not equipped with any yaw control system. A control board including motion sensors, a wireless communication device, and a microcontroller is mounted near the C.G. of the drone. A commercial electric speed controller (ESC, MX-3A, HobbyKing.com) is placed above the control board. The components are connected through four carbon fiber-reinforced plastic (CFRP) rods with a diameter of $1 \mathrm{~mm}$. The weight breakdown of the flapping drone is shown in Table 2 . The weight of the wings is only $2 \%$ of the total weight of the drone, which is the same as the corresponding ratio for a bumblebee. ${ }^{(8)}$ 
Table 1

Specifications of flapping drone.

\begin{tabular}{|c|c|c|}
\hline Parameter & Unit & Value \\
\hline Full span & $\mathrm{mm}$ & 180 \\
\hline Body height & $\mathrm{mm}$ & 140 \\
\hline Body width & $\mathrm{mm}$ & 35 \\
\hline $\begin{array}{l}\text { Flapping frequency in } \\
\text { hovering }\end{array}$ & $\mathrm{Hz}$ & 32 \\
\hline $\begin{array}{l}\text { Maximum flapping } \\
\text { frequency }\end{array}$ & $\mathrm{Hz}$ & 35 \\
\hline Maximum thrust & gf & 28 \\
\hline \multirow{3}{*}{ Center of flapping pivots ${ }^{*}$} & \multirow{3}{*}{$\mathrm{mm}$} & -0.513 \\
\hline & & 0.466 \\
\hline & & -24.0 \\
\hline \multirow{6}{*}{ Inertial moment of body ${ }^{*}$} & \multirow{6}{*}{$\mathrm{kg} \cdot \mathrm{mm}^{2}$} & 30.5 \\
\hline & & 30.3 \\
\hline & & 1.53 \\
\hline & & 0.030 \\
\hline & & -0.258 \\
\hline & & 0.067 \\
\hline
\end{tabular}

*The calculation did not include the wires, adhesive, and legs.
Table 2

Weight breakdown of flapping drone.

\begin{tabular}{|c|c|c|}
\hline Component & Weight (g) & Percentage $(\%)$ \\
\hline DC motor & 3.3 & 16.1 \\
\hline $\begin{array}{l}\text { Thorax structure } \\
\text { (Flapping mechanism) }\end{array}$ & 4.0 & 19.5 \\
\hline Wings & $0.2 \times 2$ & 2.0 \\
\hline Battery & 3.0 & 14.6 \\
\hline Control actuators & $1.54 \times 2$ & 15.0 \\
\hline $\begin{array}{l}\text { Avionics (Control/ } \\
\text { Switch boards and } \\
\text { ESC) }\end{array}$ & 4.33 & 21.1 \\
\hline $\begin{array}{l}\text { Abdomen structure } \\
\text { (Gimbal mechanism) }\end{array}$ & 0.83 & 4.0 \\
\hline $\begin{array}{l}\text { Wires, adhesive, and } \\
\text { screws }\end{array}$ & 1.2 & 5.9 \\
\hline Legs & $0.09 \times 4$ & 1.8 \\
\hline Total & 20.5 & 100 \\
\hline
\end{tabular}

\section{Flapping Mechanism}

The flapping mechanism in the thorax of the drone is illustrated in Fig. 2. The speed of the rotation produced by the DC motor is reduced by two pairs of gears with a gear ratio of 9.1:1. The rotation at the reduced speed is converted to a reciprocating motion by a novel crankslider mechanism. The crank pin and two slider pins are connected by a pair of connecting rods (named double con rods). The con rods each have a slotted hole through which each slider pin passes. The slotted holes act as a clutch in the reverse motion of the slider, in which the linear motion of the slider is disconnected from the rotation of the crank. At that moment, the slider compresses the springs placed at the ends of the slider rails. The elastic energy stored in the springs is utilized to accelerate the slider for the following stroke. The reciprocating motion of the slider is transmitted to two timing pulleys by timing belts and converted to the reciprocating flapping motion of the two wings. The slider also acts as a counterbalance against the flapping wings, which reduces the vibration generated by the flapping motion. The thorax frames and slider made of polycarbonate and the double con rods made of polyetheretherketone (PEEK) were manufactured by machining. The gears other than the crank gear are commercially available items. This flapping mechanism is accompanied by elastic deformation caused by the springs, timing belts, and thorax frame, which reduces the load of the mechanism and enhances the power efficiency. Owing to the elastic mechanism, the flapping amplitude at the wing base is dependent on the flapping frequency and wing characteristics. Figure 3 shows an example of the flapping amplitude with respect to the input flapping frequency for two of the wing models shown in Table 3. The flapping amplitude increases from 56 to 70 deg with the flapping frequency. 


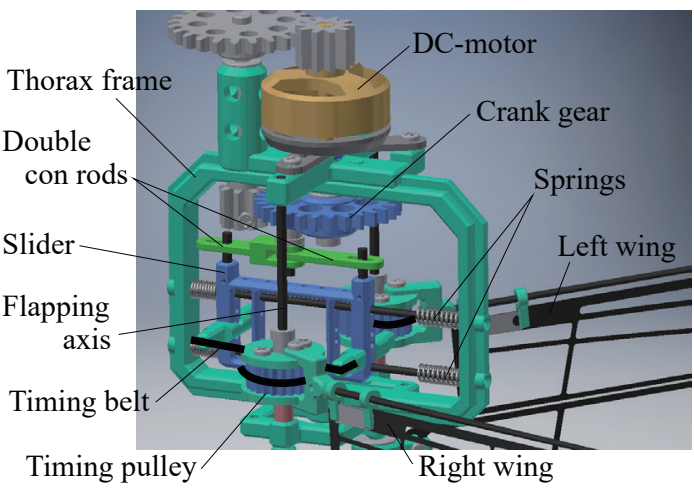

Fig. 2. (Color online) Flapping mechanism in thorax of drone.

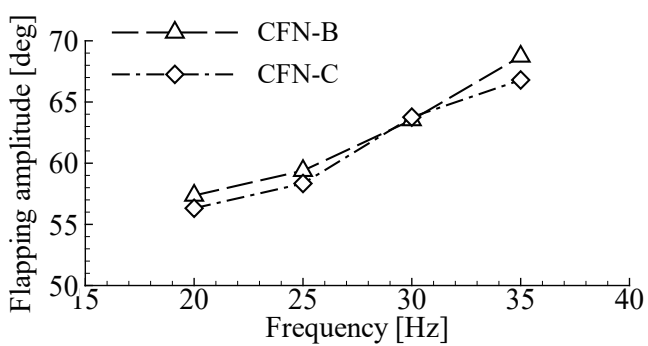

Fig. 3. Flapping amplitude at wing base with respect to input flapping frequency for two wing models shown in Table 3.

Table 3

(Color online) Specifications of developed flapping wings.

\begin{tabular}{lccccc}
\hline Name & CFN-A & CFN-B & CFN-C & CFN-D & CFN-E (present) \\
\hline Image & & & & & \\
& & & & & \\
\hline Mass & $0.17 \mathrm{~g}$ & $0.16 \mathrm{~g}$ & $0.19 \mathrm{~g}$ & $0.20 \mathrm{~g}$ & $0.20 \mathrm{~g}$ \\
\hline Span, $R$ & $82.2 \mathrm{~mm}$ & $80.6 \mathrm{~mm}$ & $82.6 \mathrm{~mm}$ & $82.2 \mathrm{~mm}$ & $82.8 \mathrm{~mm}$ \\
\hline Area & $1500 \mathrm{~mm}^{2}$ & $1180 \mathrm{~mm}^{2}$ & $1510 \mathrm{~mm}^{2}$ & $1690 \mathrm{~mm}^{2}$ & $1730 \mathrm{~mm}^{2}$ \\
\hline $\begin{array}{l}\text { CFRP } \\
\text { layers }\end{array}$ & {$[90 / 0 / 90]$} & {$[90 / 0 / 90]$} & {$[90 / 0 / 90]$} & {$[90 / 0 / 0 / 90]$} & {$[90 / 0 / 0 / 90]$} \\
\hline
\end{tabular}

\section{Flapping Wing Design}

\subsection{Wing structure}

A schematic of the structure and response of the wing during a flapping stroke is shown in Fig. 4. Although the wing structure is based on the membrane-type flapping wing developed by Keennon et al., ${ }^{(1)}$ our flapping wings are characterized by a vein pattern and positive utilization of snap-through buckling of the wing surface. The wing consists of a spar rod and a vein-reinforced membrane. The spar rod is made of a unidirectional CFRP with a diameter tapering from 0.8 to $0.5 \mathrm{~mm}$. The membrane with a thickness of $13.5 \mu \mathrm{m}$ is made of a nonwoven fabric impregnated with polyethylene terephthalate (PET). Furthermore, the membrane is reinforced by a CFRP vein pattern. The vein pattern with a line width of $0.75 \mathrm{~mm}$ was cut from an anisotropic three- or four-layer CFRP laminate with a ply thickness of $25 \mu \mathrm{m}$. The wing surface has a sleeve along the leading edge of the membrane to pass the spar rod, which allows the membrane to rotate around the spar rod. In addition, the membrane is connected to a bearing at the wing base, which is also allowed to rotate around the flapping axis. When an out-of-plane force acts on the simply supported membrane, in-plane tensile stress is produced 

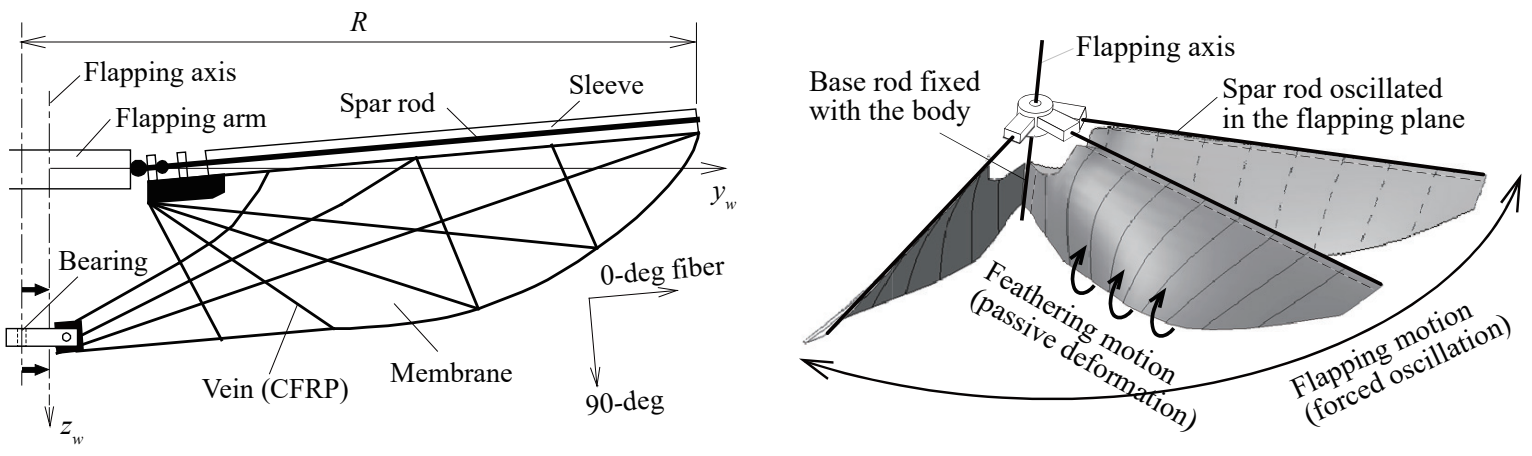

Fig. 4. Schematic wing structure and response during flapping stroke.

in the membrane, which reinforces the flexural rigidity of the membrane through the so-called stress stiffening effect. The wing base is connected to the bearing after being slightly pushed to the tip side from the natural length of the wing. Accordingly, out-of-plane buckling occurs on the wing surface because the leading edge of the membrane is not allowed to slide in the spanwise direction. When the spar rod is forcedly oscillated in the out-of-plane direction as a flapping motion, aeroelastic deformation occurs in addition to the out-of-plane buckling, which passively produces a large torsion angle (or feathering angle) during the flapping stroke. When the flapping stroke is reversed, snap-through buckling occurs on the wing surface, producing a fast feathering rotation at the stroke reversal, which enhances the aerodynamic efficiency of the flapping wing through the rotational circulation effect. ${ }^{(9-11)}$

Table 3 shows the history of the developed wings, which are named in the order of development. The latest model CFN-E is employed in the present drone. The wing planform, the vein pattern, and the stacking sequence of the CFRP laminate of the veins have been investigated with a trial-and-error approach in terms of the aerodynamic and structural characteristics of the wings and the flight stability of the drone. The weight reduction of the wing is critical to reducing the power consumption of the flapping wings. The vein patterns were determined in terms of weight reduction, aeroelastic deformation, and the strength of the wing. CFN-D and CFN-E have a slit at the center of the membrane, which mimics the connection of the fore- and hindwings of insects. It is known that some insects (e.g., bees) change the connecting angles between the fore- and hindwings and form a variable-camber airfoil during a flapping cycle, which enhances the aerodynamic efficiency. ${ }^{(12)}$ Furthermore, the planforms of CFN-D and CFN-E were improved to increase the wing base area. The large wing base area enhances the passive yaw stability of the drone because it increases aerodynamic drag against a yawing rotation of the body, although it slightly reduces the aerodynamic efficiency of the flapping wing.

\subsection{Structural characteristics in numerical simulation}

The design of the wing structure has been partly aided by numerical analysis. A finite element model for the wing structures was constructed using commercial finite element method 
(FEM) software (ANSYS 19.1, ANSYS Inc.). Four-node shell elements were used for the membrane, and beam elements were used for the leading-edge spar and veins. The anisotropy of the veins was considered on the basis of the classic composite laminate beam theory. Joint elements were used at the base of the leading edge and the wing base bearing. The contact between the sleeve and the spar was also considered. The numerical calculation is described in detail in Ref. 13.

The post-buckling deformation of the wing models was calculated in the FEM analysis. Figure 5 shows the torsion angles at the $50 \%$ span station for three of the wing models when a spanwise gradient pressure was applied to the wing surface. Figure 6 shows the deformation of the same three wing models in post-buckling with no pressure load. With increasing pressure load, the torsion angle converges to a particular angle through the stress stiffness effect. Conversely, with decreasing pressure load, snap-through occurs at a particular negative pressure load, and the torsion angle jumps to the opposite side. The three wing models have different torsion angles in post-buckling and different snap-through loads. CFN-D was designed to achieve both a large torsion angle in post-buckling and a small snap-through load.

\subsection{Aerodynamic characteristics in experiment}

The aerodynamic characteristics of the developed wing models were experimentally evaluated in terms of the mean lift-to-power ratio and wing response during a flapping cycle. In the measurement, the wing was mounted on the flapping mechanical apparatus placed on an electric balance (GX-2000, A\&D Co., Ltd.). The leading edge of the wing was directed vertically downward, and the mean lift generated downward by the flapping wing was measured with the electric balance. The mean electric power supplied to the flapping mechanical apparatus,

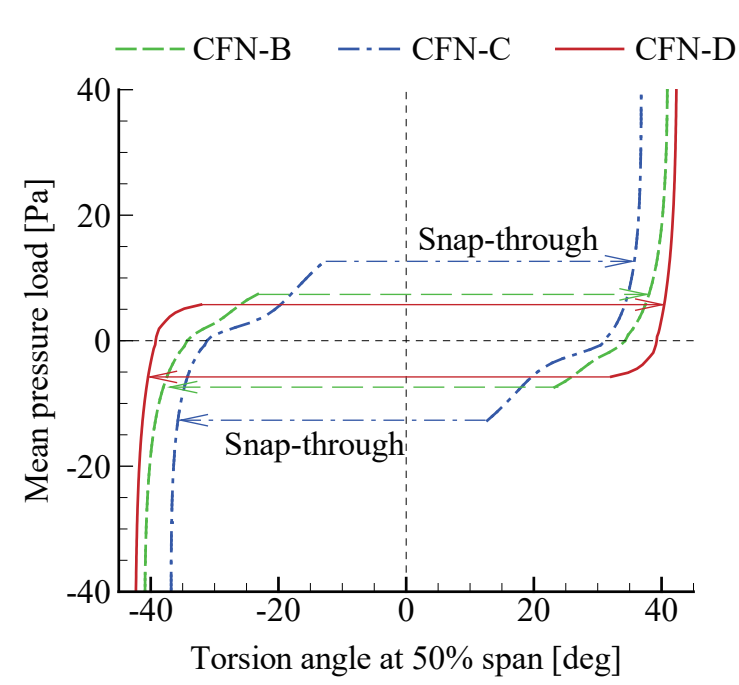

Fig. 5. (Color online) Post-buckling characteristics of three wing models with gradient pressure load.

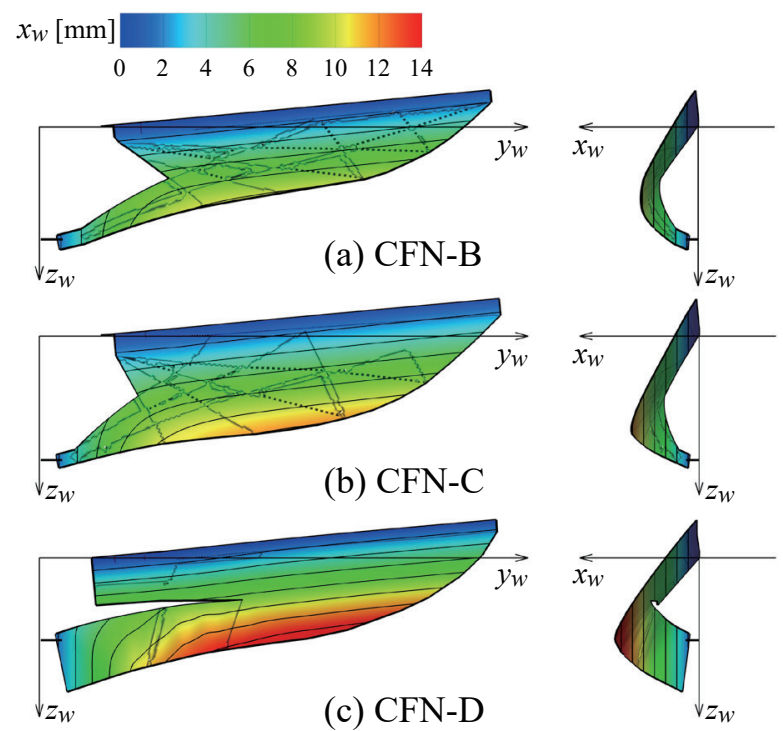

Fig. 6. (Color online) Deformation of wing models when mounted on drone with no pressure load. 
which includes the mechanical power in addition to the aerodynamic power of the wing, was also measured. At the same time, the wing response was recorded by three high-speed video cameras (FASTCAM 1024PCI, Photron Ltd.) with a frame rate of $3000 \mathrm{fps}$. Ten points on the wing surface (at the leading and trailing edges and the chord center in the $25 \%, 50 \%$, and $75 \%$ span stations in addition to the wing tip) were traced during a flapping cycle and analyzed with 3D motion analysis software (Dipp-Motion PRO V2.24a, Ditect Co., Ltd.).

Figure 7(a) shows the mean lift for a single wing with respect to the flapping frequency. The corresponding relationship between mean lift and mean power is shown in Fig. 7(b). The mean lift with respect to the flapping frequency increases from CFN-A to CFN-C. The lift-to-power ratio of CFN-D is improved by introducing a slit mimicking the fore- and hindwings of insects, although the mean lift of CFN-D is slightly smaller than that of CFN-C.

Figure 8 shows the wing responses for CFN-B, CFN-C, and CFN-D during a flapping cycle. The wing response is evaluated in terms of the flapping angle at the wing base and the feathering angles and camber ratios at $25 \%, 50 \%$, and $75 \%$ span stations. CFN-C is superior to CFN-B in terms of the camber ratio; the camber ratio of CFN-C is larger than that of CFN-B in both the up- and downstrokes, although the time histories of the feathering angles are similar. CFN-D was improved in terms of the waveforms of the feathering angles and camber ratios. The waveforms of the feathering angles and camber ratios become trapezoidal rather than sinusoidal; they are more similar to the wing motions of insects ${ }^{(8,14)}$ and enhance the aerodynamic efficiency of the flapping wing. ${ }^{(9-11)}$

The lift characteristic of the latest model CFN-E is shown in Fig. 9. The lift measurement was conducted with a pair of wings mounted on the tethered drone. As shown in Fig. 9, the flapping frequency at which the lift balances the body weight is about $32 \mathrm{~Hz}$. The maximum lift is $28 \mathrm{gf}$ at a flapping frequency of $35 \mathrm{~Hz}$ with the full throttle of the drone.

\section{Control Mechanism}

The flapping drone stabilizes its pitch and roll angles of the body by changing the gravity center position with feedback control. The gravity center position is moved by changing the mounting angles of the battery using two linear servo actuators. A schematic block diagram of the feedback control system is shown in Fig. 10. The attitude angles of the body are measured

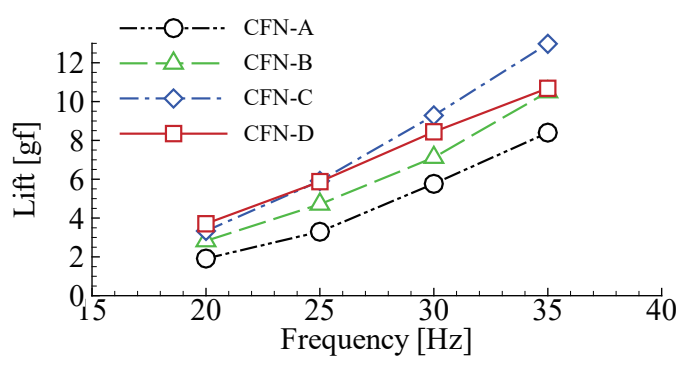

(a)

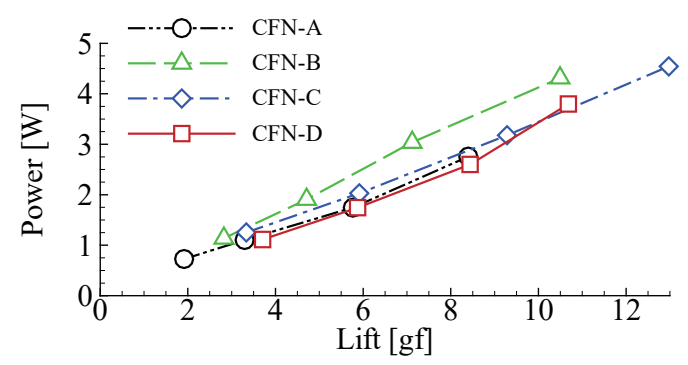

(b)

Fig. 7. (Color online) Aerodynamic characteristics of flapping wing models. (a) Mean lift vs flapping frequency. (b) Mean power vs mean lift. 

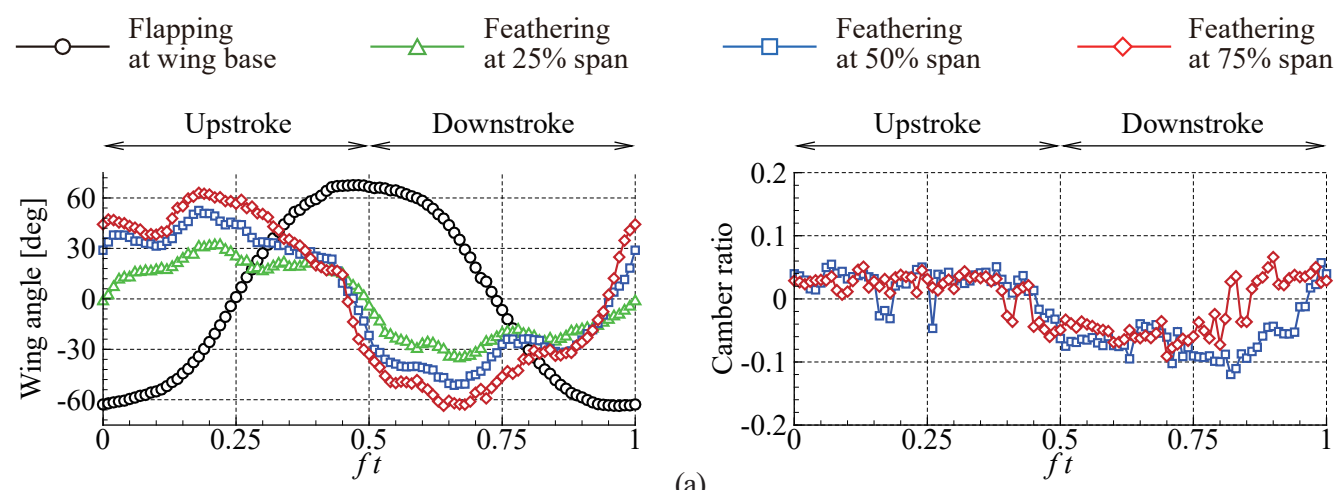

(a)
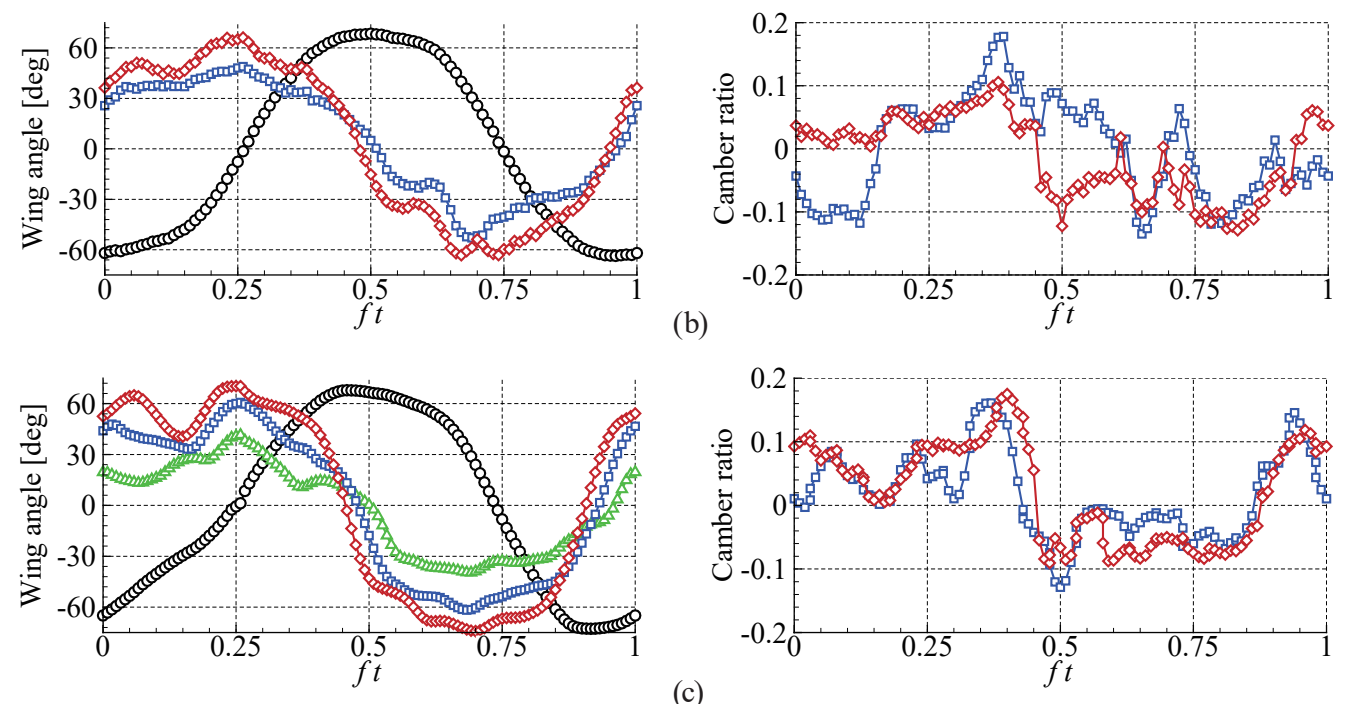

Fig. 8. (Color online) Time histories of wing deformation during flapping cycle for three wing models: (left) wing angles and (right) camber ratios. (a) CFN-B at flapping frequency of $30 \mathrm{~Hz}$. (b) CFN-C at flapping frequency of $30 \mathrm{~Hz}$. (c) CFN-D at flapping frequency of $25 \mathrm{~Hz}$.

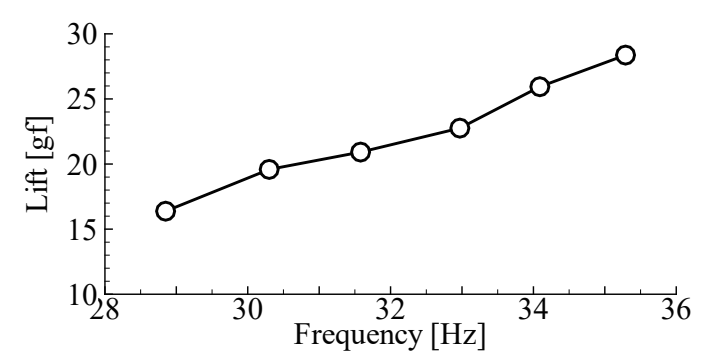

Fig. 9. Total lift generated by two wings (CFN-E) mounted on tethered flapping drone.

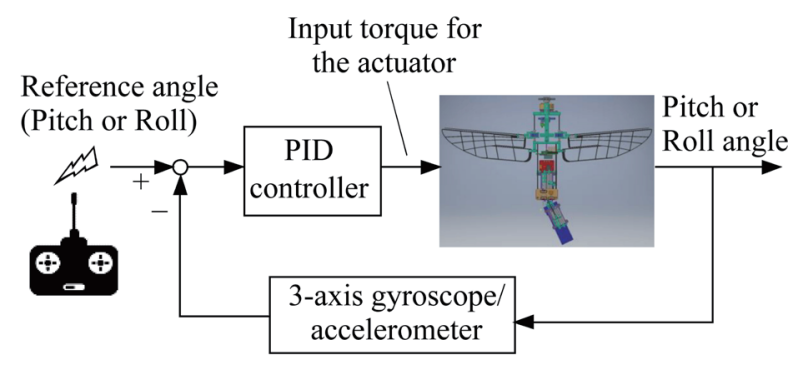

Fig. 10. (Color online) Schematic block diagram of control system.

using two motion sensors mounted on the control board: a three-axis accelerometer/gyroscope (LSM6DS3TR, STMicroelectronics) and a three-axis accelerometer (SCA3100, Murata Manufacturing Co., Ltd.). From the difference between the measured and reference pitch and roll angles, the control inputs for the two linear actuators are calculated from a proportional- 
integral-derivative (PID) control law. The control system is implemented on a microcontroller (STM32F446RET, STMicroelectronics) on the control board. The attitude angles and the throttle of the DC motor are transmitted to a Bluetooth ${ }^{\circledR}$ Low Energy module (MBN52832, Murata Manufacturing Co., Ltd.) on the control board. In addition, the outputs of the pitch and roll angles are recorded in the microcontroller and transmitted from the Bluetooth ${ }^{\circledR}$ Low Energy module to a mobile tablet device.

Figure 11 shows the gimbal mechanism used to change the mounting angles of the battery in the tail of the body. The battery can be rotated $360 \mathrm{deg}$ around the $z$-axis with the two-axis gimbal mechanism. The mounting angles of the battery are changed by two micro linear servo actuators (GS-1502, Dong Yang Model Technology Co., Ltd.). The actuator for pitch control is mounted on the battery and that for roll control is mounted on the body frame above the gimbal mechanism. The displacement of the C.G. with the two linear actuators is shown in Fig. 12. The linear actuator provides a displacement of $\pm 2.5 \mathrm{~mm}$; accordingly, the C.G. moves $\pm 2.0 \mathrm{~mm}$ in the $x$-direction for pitch control and $\pm 2.5 \mathrm{~mm}$ in the $y$-direction for roll control. The displacement velocity of the linear actuator is $40 \mathrm{~mm} / \mathrm{s}$.

\section{6. $\quad$ Flight Testing}

Using the configuration described above, autonomous flight tests of the flapping drone were conducted under wireless conditions. Note that a flexible, light string was tied at the tail as a safety harness when loss of control occurs, which did not apply any tension to the drone in flight. We succeeded in the autonomous hovering flight and vertical take-off of the flapping drone. Figure 13 shows photographs of the hovering flight and vertical take-off. Note that the
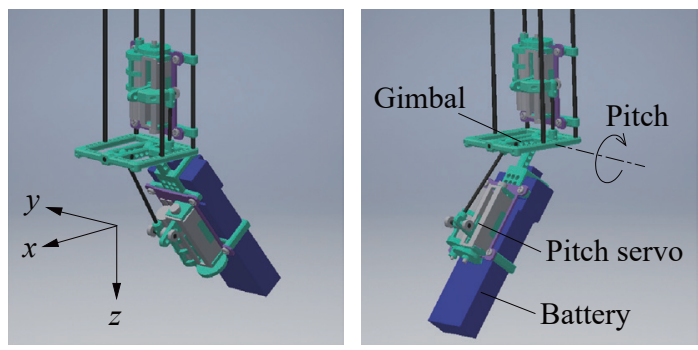

(a)

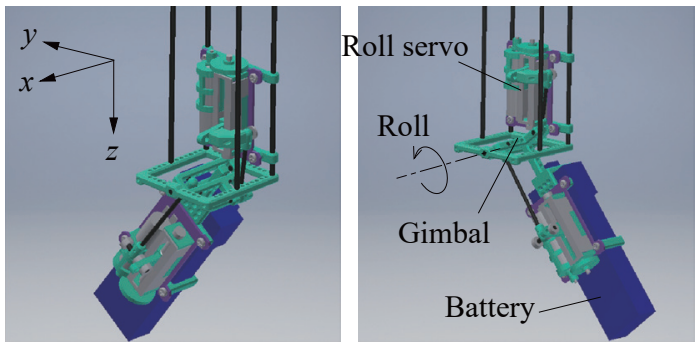

(b)

Fig. 11. (Color online) Gimbal control mechanism in abdomen of drone. (a) Pitch control. (b) Roll control.

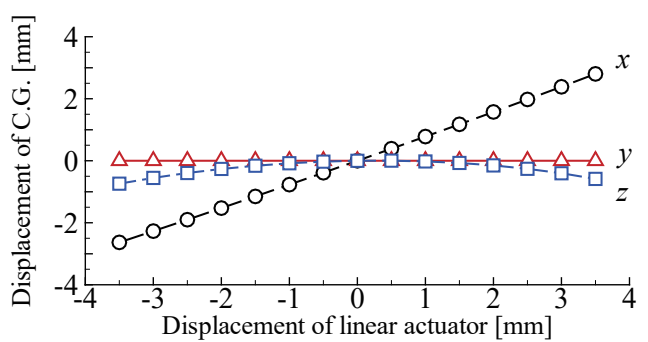

(a)

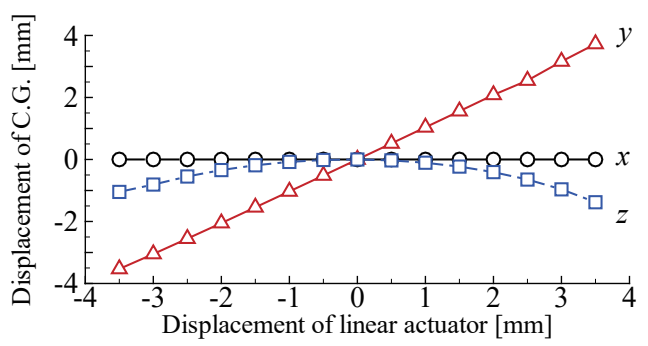

(b)

Fig. 12. (Color online) Displacement of C.G. with linear actuators. (a) Pitch control. (b) Roll control. 
four legs were removed in the hovering flight. Figure 14 shows the recorded pitch and roll angles of the body in the hovering flight, which were measured with the two motion sensors mounted on the control board. The deviations of the pitch and roll angles are within \pm 5 deg for more than $30 \mathrm{~s}$. However, the continued oscillations in both pitch and roll motions indicate that the present PID control is not optimized for the flapping drone. Figure 15 shows the frequency spectra of the pitch and roll angles in the corresponding hovering flight. There are peak frequencies at 0.9 and $1.6 \mathrm{~Hz}$ in both the pitch and roll motions of the drone. Nagai et al. ${ }^{(15)}$ and Fujita et al. ${ }^{(16)}$ solved the equation of motion in hovering flight for a flapping drone similar to the present one, which employed different wing models from CFN-E. They used a technique of stability analysis based on eigenvalues and eigenvectors of the equation of motion. They also showed that there is an unstable oscillation mode in both the pitch and roll motions at about $0.8 \mathrm{~Hz}$ in their models.

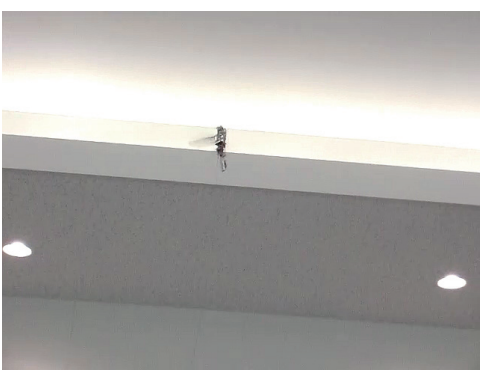

(a)

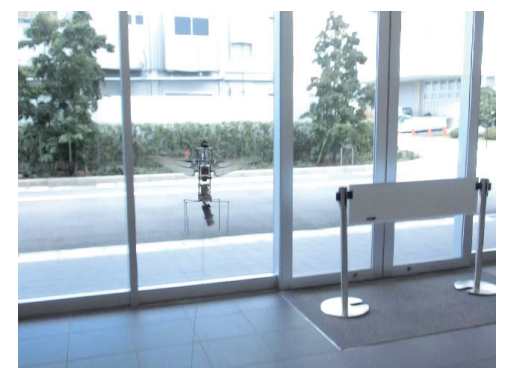

(b)

Fig. 13. (Color online) Photographs taken during wireless, autonomous flight of flapping drone. (a) Hovering flight. (b) Vertical take-off.

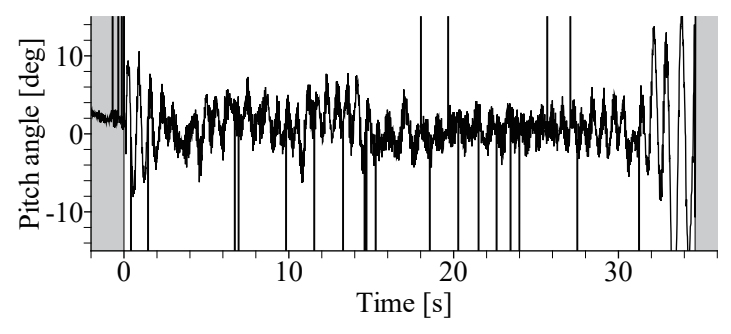

(a)

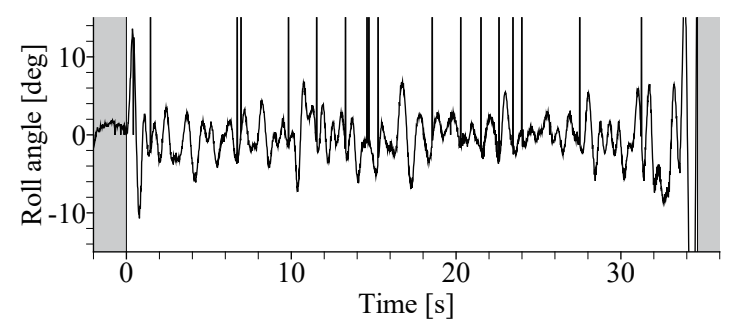

(b)

Fig. 14. Pitch and roll angles during autonomous hovering flight. (a) Pitch angle. (b) Roll angle.

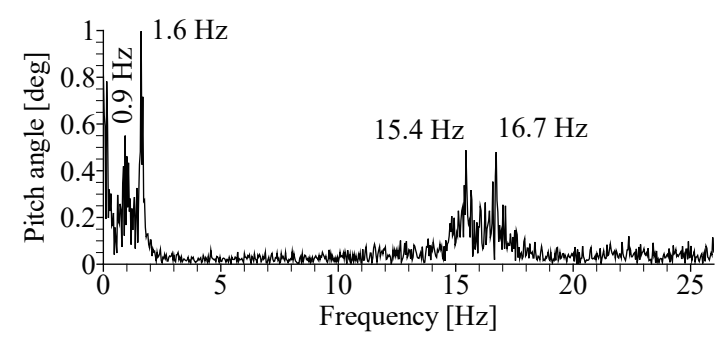

(a)

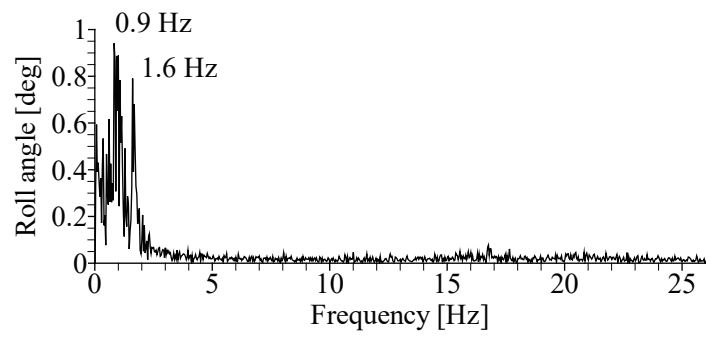

(b)

Fig. 15. Frequency spectra of pitch and roll angles during autonomous hovering flight. (a) Pitch angle. (b) Roll angle. 
Thus, the measured oscillation at $0.9 \mathrm{~Hz}$ for the present drone can be attributed to an eigenmode of the dynamics of the drone. However, the oscillation at $1.6 \mathrm{~Hz}$ has not been explained thus far. On the other hand, there are peak frequencies at 15.4 and $16.7 \mathrm{~Hz}$ only in the pitch motion, which are about half of the input flapping frequency of 31-33 Hz. Using a high-speed video camera, we confirmed that there is an oscillation mode caused by the flapping mechanism at half of the flapping frequency. Thus, the measured oscillations at 15.4 and $16.7 \mathrm{~Hz}$ can be attributed to the oscillation of the flapping mechanism, which is supported by the fact that the reciprocating motion in the flapping mechanism is directed not in the $y$-direction (roll) but in the $x$-direction (pitch). After the $30 \mathrm{~s}$ hovering flight, the body tilted over $30 \mathrm{deg}$ and lost control. The limiting tilt angle at which the body attitude is stable is about 30 deg from the vertical axis in the gravity center position control method. The previously developed flappingwing-type drones ${ }^{(1-4)}$ employed a technique of aerodynamic force modulation by changing the supports of the wing bases to control the body attitude. Our method of gravity center position control shows comparable capability in autonomous hovering flight to the counterparts. In the next step, the combination of gravity center position control with aerodynamic force control will be a key technology for the flapping drone to achieve comparable flight performance to insects and birds.

\section{Conclusions}

We have developed a tailless, two-winged flapping drone with a full span length of $180 \mathrm{~mm}$ and a total weight of $20.5 \mathrm{~g}$. A novel flapping mechanism, a control mechanism, and flapping wings have been developed for the flapping drone. The wing was designed on the basis of the experimental and numerical results in terms of aerodynamic and structural characteristics. We have succeeded in an autonomous hovering flight for more than $30 \mathrm{~s}$ and a vertical take-off for the flapping drone using the gravity center position control method. The flapping wings, the flapping and control mechanisms, and the control board were custom-made for the flapping drone, whereas commercially available items were employed for the other parts to reduce the cost. Although the current manufacturing of the structure is mainly based on machining, the cost can be reduced by plastic injection molding. To realize a widely available, nature-friendly drone, further investigation is necessary to evaluate the flight characteristics for safety. The development of flapping drones also including the previously developed ones is still dependent on an experimental approach using trial and error. In order to optimize the design of flapping drones to approach the flight performance of birds and insects, it is necessary to conduct multidisciplinary optimization considering the aerodynamics, structure, dynamics, and control of the flapping drone.

\section{Acknowledgments}

This work was supported by Murata Manufacturing Co., Ltd., and Japan Society for the Promotion of Science KAKENHI (Grant Number JP19K04838). The authors thank Shota Yonemoto for his support in the experiment. 


\section{References}

1 M. Keennon, K. Klingebiel, H. Won, and A. Andriukov: Proc. 50th AIAA Aerospace Sciences Meeting Including the New Horizons Forum and Aerospace Exposition (AIAA, 2012) AIAA-2012-0588. https://doi. org/10.2514/6.2012-588

2 A. Roshanbin, H. Altartouri, M. Karásek, and A. Preumont: Int. J. Micro Air Veh. 9 (2017) 270. https://doi. org $/ 10.1177 / 1756829317695563$

3 H. V. Phan, S. Aurecianus, T. Kang, and H. C. Park: KUBeetle-S: Int. J. Micro Air Veh. 11 (2019) 1. https://doi. org $/ 10.1177 / 1756829319861371$

4 M. Karasek, F. T. Muijres, C. De Wagter, B. D. W. Remes, and G. C. H. E. de Croon: Science 361 (2018) 1089. https://doi.org/10.1126/science.aat0350

5 A. J. Hinterwirth and T. L. Daniel: J. Comp. Physiol. A 196 (2010) 947. https://doi.org/10.1007/s00359-010$\underline{0578-5}$

6 T. Luu, A. Cheung, D. Ball, and M. V. Srinivasan: J. Exp. Biol. 214 (2011) 2215. https://doi.org/10.1242/ jeb.050310

7 J. P. Dyhr, K. A. Morgansen, T. L. Daniel, and N. J. Cowan: J. Exp. Biol. 216 (2013) 1523. https://doi. org/10.1242/jeb.077644

8 R. Dudley and C. P. Ellington: J. Exp. Biol. 148 (1990) 19.

9 M. H. Dickinson, F.-O. Lehmann, and S. P. Sane: Science 18 (1999) 1954. https://doi.org/10.1126/ science.284.5422.1954

10 S. P. Sane and M. H. Dickinson: J. Exp. Biol. 204 (2001) 2607.

11 H. Nagai, and K. Isogai: AIAA J. 49 (2011) 1750. https://doi.org/10.2514/1.J050968

12 H. Nagai, K. Isogai, N. Uda, and K. Ono: Proc 29th Congress of the International Council of the Aeronautical Sciences (ICAS, 2014) ICAS2014-4.3.3.

13 H. Nagai, K. Nakamura, M. Murozono, K. Fujita, H. Arizono, S. Nagasaki, and S. Yashiro: Proc. 2nd Int. Symp. Flutter and its Application (ISFA, 2020) 248-263.

14 A. Azuma and T. Watanabe: J. Exp. Biol. 116 (1985) 79.

15 H. Nagai, K. Nakamura, K. Fujita, M. Murozono, S. Nagasaki, and H. Arizono: Proc. 57th Aircraft Symp. JSASS (JSASS, 2019) JSASS-2019-5018 (in Japanese).

16 K. Fujita, K. Ohama, H. Nagai, M. Murozono, and K. Nakamura: Proc. JSASS Western Branch Symp. (JSASS, 2019) JSASS-2019-S002 (in Japanese).

\section{About the Authors}

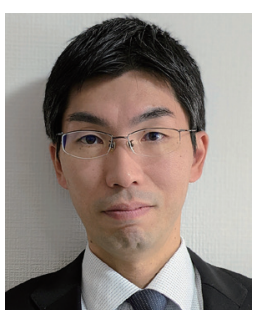

Hiroto Nagai received his B.S. and M.S. degrees in engineering from Kyushu University, Japan, in 2002 and 2004, respectively, and his Ph.D. degree in engineering from Tohoku University, Japan, in 2008. From 2009 to 2013, he was an assistant/associate professor at Nippon Bunri University, Japan. From 2013 to 2020, he was an assistant professor at Kyushu University, Japan. Since 2020, he has been an associate professor at Nagasaki University. His research interests are in aeroelasticity, vibration, aerodynamics, and micro air vehicles. (nagai.hiroto@nagasaki-u.ac.jp)

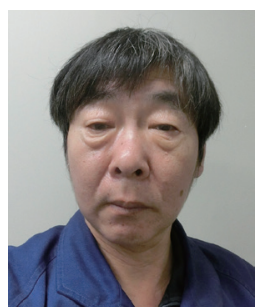

Kazutaka Nakamura received his B.S. degree in engineering from Osaka University, Japan, in 1985. Since 1985, he has engaged in the development of ceramic materials for electronic applications at Murata Manufacturing Co., Ltd. Since 2013, he has engaged in the design and development of the flapping drone. (nakamura_kazutaka@murata.com) 


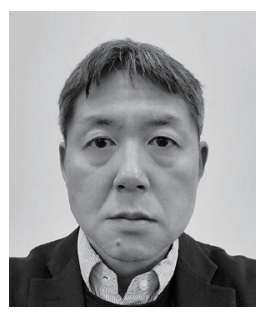

Koki Fujita received his B.S. degree from Kyushu University, Japan, in 1993 and his M.S. and Ph.D. degrees from Kyushu University, Japan, in 1995 and 1998, respectively. From 1999 to 2017, he was an assistant professor at Kyushu University, Japan. Since 2017, he has been an associate professor at Nippon Bunri University. His research interests are in intelligent sensing and control systems for aerospace engineering, especially in the guidance and control of space robots, as well as in the modeling of the space debris environment. (fujitakk@nbu.ac.jp)

Issei Tanaka received his B.S. and M.S. degrees in engineering from Kyushu University, Japan, in 2017 and 2019, respectively. Since 2019, he has been working for Nissan Motor Co., Ltd.

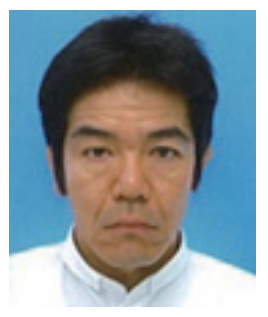

Shuji Nagasaki received his B.S. and M.S. degrees in engineering from Kyushu University, Japan, in 1993 and 1995, respectively. Since 1998, he has been an assistant professor at Kyushu University, Japan. His research interests are in the flight dynamics and design of small UAVs, as well as in the design of multi-rendezvous trajectories for active space debris removal. (nagasaki@aero.kyushu-u.ac.jp)

Yutaka Kinjo received his B.S. degree in engineering from the University of the Ryukyus, Japan, in 2011. Since 2011, he has been a technical staff specializing in electronics and mechatronics at Kyushu University.

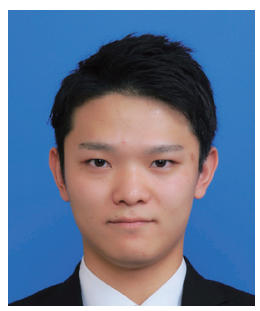

Shintaro Kuwazono received his B.S. degree in engineering from Nagasaki University, Japan, in 2020. He is currently a Master's student at the Graduate School of Engineering of Nagasaki University, Japan. His research interests are in mechanical vibration and micro air vehicles.

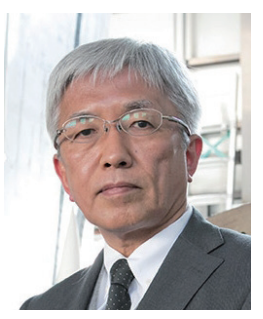

Masahiko Murozono received his B.S., M.S., and Ph.D. degrees in engineering from Kyushu University, Japan, in 1981, 1983, and 1986, respectively. From 1986 to 2016, he was an assistant/associate professor at Kyushu University, Japan. Since 2016, he has been a professor at Nippon Bunri University. His research interests are in thermoelasticity, the dynamics of thin-walled structures, and mechanical and structural vibrations. (murozonomh@nbu.ac.jp) 